\title{
Structural studies of membrane- associated proteins and molecular machines by cryo-electron microscopy
}

Alok K. Mitra, ${ }^{*}$, Herve Celia ${ }^{1}$, Gang Ren ${ }^{2}$, Luc Teyton $^{3}$, Joel Quispe ${ }^{4}$, Stephen H. Leppla ${ }^{5}$, Suzanne Manning ${ }^{6}$, Srdjan Mitrovic ${ }^{6}$, Susan Turner ${ }^{7}$, Sarah Greig ${ }^{6}$, Judy O’Brien ${ }^{8}$, John Taylor ${ }^{8}$ and Richard Bellamy ${ }^{8}$.

*Electron Crystallography Laboratory, School of Biological Sciences, University of Auckland, Private Bag 92019, Auckland, NZ

${ }^{1}$ University of Strassbourg, F67412 Illkirch, Cedex France

${ }^{2}$ Dept. of Biochemsitry and Molecular Biology, Baylor College of Medicine, Houston, TX.

${ }^{3}$ Dept. of Immunology, The Scripps Research Institute, La Jolla, CA.

${ }^{4}$ Dept. of Cell Biology, The Scripps Research Institute, La Jolla, CA.

${ }^{5}$ NIAID, NIH, 9000 Rockville Pike, Bethesda, MD.

${ }^{6}$ Electron Crystallography Laboratory, School of Biological Sciences, University of Auckland, Private Bag 92019, Auckland, NZ

${ }^{7}$ Microbiology Research Group, School of Biological Sciences, University of Auckland, Private Bag 92019, Auckland, NZ

${ }^{8}$ Virology Research Group, School of Biological Sciences, University of Auckland, Private Bag 92019, Auckland, NZ

Our research interest is to understand structure/function relationships of membrane proteins, membrane-associated proteins and large macromolecular complexes of biomedical importance. These systems, usually refractory to structural analysis by most methods, are ideally suited for high-resolution investigation using electron cryo-microscopy. We describe our findings on four systems as follows that will be discussed in full detail.

1) Structural studies of cellular immune receptors such as MHC molecules, T cell receptor (TCR) and TCR/MHC complexes have been carried out with recombinant soluble forms of the extra cytoplasmic domain of these glycoproteins. The important role of the membrane bilayer in $\mathrm{T}$ cell recognition and antigen presentation has become increasingly obvious with the description of lipid micro domains where these molecules segregate. However, the interactions and orientation of these receptors at the lipid bilayer are unknown. We have used $\mathrm{H}-2 \mathrm{~K}^{\mathrm{b}}$, a major-histocompatibility (MHC) class I molecule, and tethered its soluble domain to a lipid bilayer via a surrogate connecting peptide to reveal the disposition of MHC molecule on the membrane surface. We demonstrate that the long axis of the MHC molecule is approximately parallel to the plane of the membrane with the peptide-binding pocket close to the membrane surface. This orientation is orthogonal to the commonly held textbook depiction and potentially has implications in the important interactions with accessory molecules. This result was determined by analysing $4.5 \AA$ resolution electron crystallographic projection data from frozenhydrated 2-dimensional crystals. Ionic interactions between the lipid head group and the protein appear to be responsible for this orientation.

2) Anthrax toxin (AT), secreted by Bacillus anthracis, is a three-protein cocktail of lethal factor (LF, $90 \mathrm{kD})$, edema factor $(\mathrm{EF}, 89 \mathrm{kD})$ and the protective antigen (PA, $83 \mathrm{kD})$. PA is a member of a unique family of soluble proteins that form channels in lipid bilayer membranes. Steps in 
anthrax toxicity involve, a) binding of ligand (EF/LF) to a heptamer of PA63 (PA63h) generated after N-terminal proteolytic cleavage of PA and, b) following endocytosis of the complex, translocation of the ligand into the cytosol by an as yet unknown mechanism. The PA63h.LF complex was directly visualized from analysis of images of specimens suspended in vitrified buffer by cryo-electron microscopy, which revealed that the LF molecule, localized to the non-membrane interacting face of the oligomer, interacts with four successive PA63 monomers and partially unravels the heptamer, thereby widening the central lumen. The observed structural reorganization in PA63h likely facilitates the passage of the large $90 \mathrm{kD} \mathrm{LF}$ molecule through the lumen en route to its eventual delivery across the membrane bilayer. The 3 -D density map of the complex determined at $\sim 18 \AA$ resolution is utilized to model the interaction of the LF molecule to the PA63 heptamer.

3) A strain of bacteria proliferating in sludge and belonging to the $\beta$ protobacteria, has been shown to produce pili-like appendages that have unusually large diameter of $\sim 500 \AA$. These appendages are generated only when the bacteria are grown in non-agitated conditions and appear to be helically ordered assemblies of membrane associated protein or proteins with a major component of molecular weight of $\sim 130 \mathrm{kD}$. The exact biological function of these pililike structures is unknown. The pili-like structures appear to be membranous sheaths encasing along their helical axis continuous or discreet array of "cargo" of unknown chemical nature, and may represent a conduit for macro-scale bacterial communication. We have recorded images of these structures while suspended in vitrified buffer and applied helical image processing to reveal the architecture of the assembly at $\sim 25 \AA$ resolution. Structural analysis is also being carried out on $2-\mathrm{D}$ crystals that are formed by solubilizing the pili in detergents such as octyl- $\beta$ D-glucoside (OG), CHAPS followed by reconstitution through removal of detergent by dialysis. Our goal is to have comprehensive picture of the full assembly and structure/function relationship of the helical assembly and the "cargo" carried inside it.

4) The rotavirus double-layered particle (DLP) is a molecular machine that transcribes 11 segments of the double-stranded RNA genome into full-length mRNA segments during viral replication. We observed that human Wa DLPs belonging to SG II possess a markedly reduced level of transcriptase activity when compared to UK bovine DLPs belonging to SG I. Threedimensional cryo-electron microscopic reconstructions (at $\sim 26 \AA$ resolution) of human Wa DLPs revealed a marked absence of VP6 trimers at the five positions surrounding the type I channel as compared to UK bovine DLPs. Taken together, these results suggest that the absence of the pentonal VP6 trimers leads to a lowered rate of RNA production for SG II DLPs. In order to determine whether the distinctive differences in the VP6 capsid layer conferred subgroup-specificity in the two types of DLPs, three-dimensional reconstructions of DLPs decorated with Fab fragments derived from subgroup-specific mAbs were undertaken. Docking of the X-ray structures of VP6 and a generic Fab fragment into the cryo-EM density maps enabled us to delineate at "pseudo atomic" resolution the dispositions of the amino acid residues defining the subgroup-specific epitopes. 